\title{
Prevalence of and risk factors for MRSA colonization in HIV-positive outpatients in Singapore
}

Win Mar Kyaw ${ }^{1 *}$, Linda Kay Lee ${ }^{1}$, Wong Chia Siong², Angela Chow Li Ping ${ }^{1,2,3}$, Brenda Ang ${ }^{4}$ and Yee Sin Leo ${ }^{4,5}$

\begin{abstract}
Background: Whilst there have been studies on the risks and outcomes of MRSA colonization and infections in HIV-positive patients, local data is limited on the risk factors for MRSA colonization among these patients. We undertook this study in a tertiary HIV care centre to document the risk factors for colonization and to determine the prevalence of MRSA colonization among HIV-positive outpatients in Singapore.

Methods: This was a cross-sectional study in which factors associated with MRSA positivity among patients with HIV infection were evaluated. A set of standardized questionnaire and data collection forms were available to interview all recruited patients. Following the interview, trained nurses collected swabs from the anterior nares/axilla/groin (NAG), throat and peri-anal regions. Information on demographics, clinical history, laboratory results and hospitalization history were retrieved from medical records.

Results: MRSA was detected in swab cultures from at least 1 site in 15 patients (5.1\%). Inclusion of throat and/or peri-anal swabs increased the sensitivity of NAG screening by $20 \%$. Predictors for MRSA colonization among HIV-positive patients were age, history of pneumonia, lymphoma, presence of a percutaneous device within the past 12 months, history of household members hospitalized more than two times within the past 12 months, and a most recent CD4 count less than 200.

Conclusions: This study highlights that a proportion of MRSA carriers would have been undetected without multiple-site screening cultures. This study could shed insight into identifying patients at risk of MRSA colonization upon hospital visit and this may suggest that a risk factor-based approach for MRSA surveillance focusing on high risk populations could be considered.
\end{abstract}

Keywords: Methicillin-resistant Staphylococcus auerus, HIV, Risk factors, Colonization

\section{Background}

Methicillin-resistant Staphylococcus auerus (MRSA) infections present a significant burden in terms of morbidity, length of hospitalization, and rising healthcare costs [1-3]. Studies have shown that staphylococcal colonization is a predominant risk factor for subsequent infection, and nasal carriage of Staphylococcus aureus increases the risk of subsequent infection two- to twelve-fold, especially during hospitalization [4-7]. Risk factors associated with MRSA colonization include

\footnotetext{
*Correspondence: mar_kyaw_win@ttsh.com.sg

${ }^{1}$ Department of Clinical Epidemiology, Communicable Disease Centre, Tan Tock Seng Hospital, 11 Jalan Tan Tock Seng, Singapore 308433, Singapore Full list of author information is available at the end of the article
}

frequent exposure to healthcare settings, previous MRSA infections, and frequent antibiotic usage [8-13].

HIV-positive patients are at higher risk of MRSA colonization associated with frequent exposure to healthcare facilities, frequent oral antibiotic intake, low CD4 count, and other behavioral risk factors [13-15]. HIV has been recognized as an independent risk factor for colonization with MRSA [16], and S. aureus infections are known to be responsible for substantial morbidity and mortality in HIV-positive patients $[17,18]$.

Whilst there have been studies on the risks and outcomes of MRSA colonization in HIV-positive patients, local data is limited on the risk factors for MRSA colonization among these patients. We undertook this

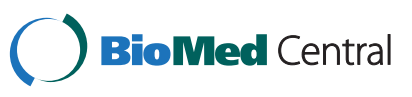


study in a tertiary HIV care centre to document the risk factors for colonization and to determine the prevalence of MRSA colonization among HIV-positive outpatients.

\section{Methods}

\section{Study type and study population}

This was a cross-sectional study in which factors associated with MRSA positivity among patients with HIV infection were evaluated. The study was done at the Communicable Disease Centre (CDC) at Tan Tock Seng Hospital (TTSH), the national referral centre for HIV. CDC is administratively responsible to TTSH, the second largest general hospital in Singapore, with 1,200beds. All HIV-positive patients who attended the specialist outpatient clinic at CDC between $12^{\text {th }}$ November and $1^{\text {st }}$ December 2008 were asked to participate. Verbal consent was obtained from all participants.

\section{Data sources and data collection}

A set of standardized questionnaire and data collection forms were available to interview all recruited patients. Information collected include visits to a healthcare facility in the past 12 months, contact with healthcare workers $(\mathrm{HCW})$ in the past 6 months, hospitalization history of household members, presence of a percutaneous device in the past 12 months or urinary catheter in the past 6 months; surgery in the past 12 months, and use of intravenous and oral antibiotics in the past 1 month and 3 months, respectively. This information was collected through interviews by a research staff.

Following the interview, trained nurses collected swabs from the anterior nares, axilla, groin, throat, and perianal regions. The first three areas were swabbed using a single swab stick. Separate swab sticks were used for the throat and peri-anal region. Samples were inoculated on chromogenic agar plates (MRSASelect, BioRad, France) and incubated at $37^{\circ} \mathrm{C}$ for $18-28$ hours. Growth of pink or mauve colonies was read as MRSA positive, while colorless colonies were MRSA negative.

Information on demographics, clinical history, laboratory results and hospitalization history were retrieved from medical records. These included the presence of

Table 1 Sites of Methicillin-resistant Staphylococcus aureus (MRSA) colonization

\begin{tabular}{lc}
\hline Site & MRSA Position Cases $[\mathbf{n}=\mathbf{1 5}]$ \\
\hline NAG only & 6 \\
Throat only & 2 \\
Peri-anal only & 1 \\
NAG + Throat & 2 \\
NAG + Peri-anal & 1 \\
NAG + Throat + Peri-anal & 3 \\
\hline
\end{tabular}

NAG $=$ Nares, axilla, groin. co-morbidities, admission to intensive care unit or general hospitalization in the past 12 months, use of antiretroviral (ART) therapy during the preceding 12 months, and current usage of co-trimoxazole and beta lactam antibiotics in the past six months. The most recent CD4 count during the preceding 6 months and previous MRSA colonization history were also collected.

\section{Statistical analysis}

For this prospective case control study, cases were defined as HIV-positive patients with laboratoryconfirmed MRSA colonization, and controls as HIVpositive patients without laboratory-confirmed MRSA colonization within the study period.

Cases and controls were compared by univariate analysis to determine differences in the allocation of potential risk factors. Descriptive statistics were used to describe the distribution of the variables in the study population. A chi square test or Fisher's exact test was used to evaluate differences in categorical variables. Crude odds ratios for continuous variables were obtained by using logistic regression. All variables with $\mathrm{p}<0.05$ in univariate analysis were included in multivariate analysis to identify independent risk factors of MRSA. A stepwise logistic regression model was used to select variables for inclusion in the final model. Variables with collinearity were not simultaneously considered in the final model.

\section{Study approval}

This study was approved by the domain specific review board (DSRB) of the National Healthcare Group, Singapore (DSRB Reference No. 2009/00545).

\section{Results}

\section{Descriptive statistics and univariate analysis}

A total of 296 HIV-positive patients were recruited, giving us a response rate of $93 \%$. They were predominantly male $(n=257,86.8 \%)$, and the median age of these patients was 43 years (range, 18-73). Among the 296 patients, MRSA was detected in swab cultures from at least 1 site in 15 patients (5.1\%). Out of these 15 patients, 6 had positive MRSA cultures from their nasal/ axilla/groin swab only while 3 were positive in either throat or peri-anal swabs only (Table 1). The demographic characteristics of the 15 cases and 281 controls are shown in Table 2 . There was a slight male predominance among the case patients; males were 2.2 times more likely than females to have MRSA.

Significant HIV-associated conditions among cases included Pneumocystis jiroveci pneumonia (PCP) and bacterial pneumonia. Patients with PCP were more likely to have MRSA than those without (odds ratio [OR], 3.3; 95\%CI, 1.14-9.30); those with bacterial pneumonia were 
Table 2 Risk factors for MRSA colonization: Univariate analysis

\begin{tabular}{|c|c|c|c|c|}
\hline Vañable & MRSA Position n (\%)* & MRSA Negatve $n(\%)^{*}$ & OR $(95 \% \mathrm{Cl})$ & $P$ value \\
\hline \multicolumn{5}{|l|}{ Demographics } \\
\hline Age(median, range), years & $50(30-67)$ & $43(18-73)$ & $1.1(1.00-1.11)$ & 0.036 \\
\hline Age $>=65$ & $1(6.7)$ & $7(2.5)$ & $2.8(0.32-24.32)$ & 0.352 \\
\hline Gender, Male & $14(933)$ & $243(86.5)$ & $2.2(0.28-17.13)$ & 0.455 \\
\hline \multicolumn{5}{|l|}{ AIDS-related infections } \\
\hline Tuberculosis & $3(20.0)$ & $46(16.4)$ & $1.3(0.35-4.71)$ & 0.713 \\
\hline Pneumoncystic jiroveci pneumonia & $8(53.3)$ & $73(26.0)$ & $3.3(1.14-9.30)$ & 0.027 \\
\hline Bacterial Pneumonia & $4(26.7)$ & $24(8.5)$ & $3.9(1.15-13.17)$ & 0.029 \\
\hline Cytomegalovirus infection & $3(20.0)$ & $25(8.9)$ & $2.6(0.68-9.68)$ & 0.166 \\
\hline Skinlesion & $6(40.0)$ & $70(24.9)$ & $2.0(0.69-5.85)$ & 0.200 \\
\hline Lymphoma & $3(20.0)$ & $4(1.4)$ & $17.3(3.48-86.15)$ & $<0.001$ \\
\hline Toxoplasmosis & $1(6.7)$ & $6(2.1)$ & $3.3(0.37-29.08)$ & 0.287 \\
\hline \multicolumn{5}{|l|}{ Exposure to Health Facilities } \\
\hline Prior hospitalization in the past one year & $9(60)$ & $44(15.7)$ & $81(274-23.84)$ & $<0.001$ \\
\hline Outpatient visit to $C D C$ in the past one year & $15(100)$ & $259(92.2)$ & NC & 0.260 \\
\hline Admission to Intensive care unit in the past one year & $1(6.7)$ & $1(0.4)$ & $20.0(1.19-336.64)$ & 0.038 \\
\hline Polyclinic visit in the past one year* * & $7(46.7)$ & $125(44.5)$ & $1.1(0.39-3.09)$ & 0.870 \\
\hline Surgery in the past one year* * & $1(6.7)$ & $11(3.9)$ & $1.8(0.21-14.55)$ & 0.603 \\
\hline Presence of Percutaneous Device in the past one year** & $11(73.3)$ & $47(16.7)$ & $13.7(4.18-44.84$ & $<0.001$ \\
\hline Urinary catheterization in the past six months* * & $1(6.7)$ & $4(1.4)$ & $5.0(0.52-47.22)$ & 0.165 \\
\hline $\begin{array}{l}\text { Household member hospitalized } \\
\text { more than two times in the past one year ** }\end{array}$ & $5(33.3)$ & $28(10.0)$ & $4.5(1.44-14.16)$ & 0.010 \\
\hline $\begin{array}{l}\text { Patient contact with a health care } \\
\text { worker in the past } 6 \text { month ** }\end{array}$ & $5(33.3)$ & $29(10.3)$ & $4.3(1.39-13.59)$ & 0.012 \\
\hline \multicolumn{5}{|l|}{ Drug history } \\
\hline Recent intravenous antibiotic usage & $8(72.7)$ & $37(78.7)$ & $0.7(0.16-3.23)$ & 0.669 \\
\hline Oral antibiotic usage in the past three months & $11(73.3)$ & $96(34.2)$ & $5.3(1.64-17.09)$ & 0.005 \\
\hline On antiretroviral therapy in the preceding one year & $14(93.3)$ & $238(84.7)$ & $2.5(0.32-17.74)$ & 0.376 \\
\hline Current use of Co-trimoxazole & $4(26.7)$ & $77(27.4)$ & $1.0(0.29-3.12)$ & 0.950 \\
\hline Beta lactam antibiotic usage in the past six months & $5(33.3)$ & $10(3.6)$ & $13.6(3.90-47.07)$ & $<0.001$ \\
\hline \multicolumn{5}{|l|}{ Others } \\
\hline History of previous MRSA colonization & $2(13.3)$ & $7(2.5)$ & $6.0(1.14-31.89)$ & 0.035 \\
\hline Most recent $C D 4<200$ & $9(60)$ & $71(25.3)$ & $4.4(1.53-12.90)$ & 0.006 \\
\hline
\end{tabular}

*Unless otherwise indicated.

* * Data collected by questionnaire.

$\mathrm{NC}=$ not calculable.

more likely to have MRSA as well $(\mathrm{OR}=3.9$; 95\%CI, 1.1513.17). Patients with lymphoma were 17.3 times more likely than patients without lymphoma to be colonized with MRSA (95\%CI, 3.48-86.15).

Among MRSA positive patients, 9 (60\%) had prior hospitalization in the past year compared with 44 (15.7\%) of the MRSA negative controls. HIV-positive patients who had outpatient visit(s) to CDC or a polyclinic within the past year were more likely to be colonized with MRSA compared to those who did not.
However, this was not statistically significant. Additionally, those who had a percutaneous device in the past 12 months, a history of household members hospitalized more than two times in the past 12 months, or contact with a health care worker in a hospital in the past 6 months were significantly more likely to have MRSA than patients without these exposures (Table 2).

Risk factors relating to drug exposure were also evaluated in Table 2. Patients with oral antibiotic use during the last 3 months were 5.3 times more likely than 
Table 3 Independent Risk Factors Associated with MRSA colonization: Multivariate analysis

\begin{tabular}{lcc}
\hline Expore & OR (95\% Cl) & P value \\
\hline Lymphoma & $15.7(2.0-123.10)$ & 0.009 \\
Most recent CD4<200 & $9.1(1.187-44.53)$ & 0.006 \\
$\begin{array}{l}\text { Hoousehold member } \\
\text { hospitalized more than }\end{array}$ & $8.4(1.80-39.18)$ & 0.007 \\
two times in the past one year & & \\
$\begin{array}{l}\text { Presence of Percutaneous } \\
\text { Device in the past one year }\end{array}$ & $8.1(2.07-31.81)$ & 0.003 \\
$\begin{array}{l}\text { Pneumonia } \\
\text { Age (years) }\end{array}$ & $6.5(1.30-32.55)$ & 0.023 \\
\hline
\end{tabular}

patients without recent oral antibiotic usage to have MRSA (73.3\% vs. 34.2\%; 95\%CI, 1.64-17.09). In addition, patients who used beta lactam antibiotics within the last 6 months were also more likely to be cases than patients who did not. Patients with a recent CD4 count less than 200 were 4.4 times more likely than patients with a recent CD4 greater than 200 to be cases than controls.

\section{Multivariate analysis}

Upon multivariate analysis, predictors for MRSA colonization among HIV-positive patients were age, history of bacterial pneumonia, lymphoma, presence of a percutaneous device within the past 12 months, history of household members hospitalized more than two times within the past 12 months, and a most recent CD4 count less than 200 (Table 3). We generated a receiver operating characteristic (ROC) curve to evaluate the final logistic regression model. The area under the ROC curve (AUC) was 0.89 (95\% CI, 0.83-0.97).

\section{Discussion}

Singapore first isolated MRSA in the early 1980s [19]. The incidence and prevalence of MRSA infections have remained steady during the last two decades in major academic medical centers, although methicillin resistance appeared in $35 \%$ of all S. aureus isolates in one study [20]. The incidence of HIV infection in Singapore has increased rapidly over the last decade [21].

A previous study at CDC showed that the prevalence of MRSA colonization in HIV-positive outpatients was higher than the prevalence of MRSA colonization in other populations [15]. A study by Villacian et al. using only nasal swab reported a lower point prevalence of MRSA colonization (3\% vs. $5.1 \%$ in our study) and $20 \%$ colonization with methicillin-sensitive Staphylococcus aureus (MSSA) [15]. Most studies and screening programs evaluating the prevalence of MRSA colonization have utilized swabs from the nares, axilla and groin $[22,23]$. Mertz et al. have shown that the inclusion of a throat swab increased the sensitivity of detection among S. aureus carriers by $25.7 \%$ [22].

In this study, we showed that inclusion of throat and/ or peri-anal swabs increased the sensitivity of NAG screening by $20 \%$. The inclusion of additional swabs could be especially important for HIV positive patients, as unrecognized carriers can serve as reservoirs for transmission during frequent hospital visits. Chow et al. found that the inclusion of additional perianal and throat swabs increased MRSA detection by $12.5 \%$ in HIV positive patients [24].

Our finding that recent diagnosis of lymphoma was associated with MRSA colonization could be explained by the increased likelihood for lymphoma in advanced HIV infection when there is a greater extent of immunosuppression [25-27]. Some studies have shown an association between MRSA colonization and cutaneous T-cell lymphoma but not among HIV patients [28,29]. Similarly, we identified low CD4 count as a risk factor for MRSA colonization; this has been described in other studies among HIV-positive patients [13,30,31].

Although univariate analysis showed an association between MRSA colonization and hospitalization within the past year, this effect was negated in multivariate analysis. Recent hospitalization has been frequently described as a predictor for MRSA colonization among HIV-positive patients in the published literature $[14,15]$. Interestingly, in this study, we found a strong association between MRSA colonization and previous hospitalization history of household members. Studies have shown that family members of colonized patients are at risk for MRSA colonization and subsequent MRSA infection for many months $[7,32,33]$.

The association between MRSA colonization and the presence of a percutaneous device within the past year in this study was significant. Onorato and colleagues found a seven-fold increased risk between the insertion of a central venous catheter and MRSA colonization among HIV-positive patients [14]. Both findings highlight the importance of aseptic insertion and appropriate care of such devices and may reflect that patients with indwelling devices could have more contacts with healthcare workers, making them more susceptible to MRSA.

Our observation that receipt of ARV within the past year was not associated with decreased risk of MRSA colonization is inconsistent with previous studies [31]. We found strong association between age and MRSA colonization. Studies have shown that elderly age (>65 years) is a risk factor for MRSA colonization despite patient group [24,34]. AUC value in our study showed that predictors in the final model comprising six variables can differentiate well between MRSA colonizers and non-colonizers. 
Our study had some limitations. Our population was predominantly male, and the sample size was small; more independent risk factors may have been identified with a larger study population. As our data were collected at a single public hospital for only outpatients, it may not reflect all HIV-infected patients. We may have underestimated prior hospitalizations, as data collection for this study did not account for admission to other hospitals. Data collection was also limited for some risk factors previously associated with MRSA colonization in HIV infected patients such as prior incarceration and high risk sexual behaviors [35,36]. Many healthcare related factors assessed by the questionnaire in this study may be subjected to recall and reporting bias. However, this study highlights that a proportion of MRSA carriers would have been undetected without multiple-site screening cultures. HIV-positive patients are at increased risk of MRSA colonization due to immunosuppression and also their exposure to healthcare facilities.

\section{Conclusions}

This study highlights that a proportion of MRSA carriers would have been undetected without multiple-site screening cultures. This study could shed insight into identifying patients at risk of MRSA colonization upon hospital visit. Predictors for MRSA colonization among HIV-positive patients were age, history of bacterial pneumonia, lymphoma, presence of a percutaneous device within the past 12 months, history of household members hospitalized more than two times within the past 12 months, and a most recent CD4 count less than 200. This may suggest that a riskfactor based approach for MRSA surveillance focusing on high risk populations could be considered.

\section{Study approval}

This study was approved by the domain specific review board (DSRB) of the National Healthcare Group, Singapore (DSRB Reference No. 2009/00545).

\section{Abbreviations \\ ARV: Antiretroviral; AUC: Area under the ROC curve; CDC: Communicable Disease Centre; HCW: Healthcare workers; MRSA: Methicillin-resistant Staphylococcus aureus; MSSA: Methicillin-sensitive Staphylococcus aureus; PCP: Pneumocystis jiroveci pneumonia; TTSH: Tan Tock Seng Hospital.}

\section{Competing interests}

The authors declare that they have no competing interests.

\section{Authors' contributions}

ACLP participated in study design and data acquisition. LKL, WCS, YSL and BA participated in manuscript writing. WMK performed data acquisition, data analysis and manuscript writing. All authors read and approved the final manuscript.

\section{Acknowledgements}

We thank Upendra and Bernard Tan from the Communicable Disease Centre who helped with patient recruitment and data collection.

\section{Author details}

'Department of Clinical Epidemiology, Communicable Disease Centre, Tan Tock Seng Hospital, 11 Jalan Tan Tock Seng, Singapore 308433, Singapore. ${ }^{2}$ Saw Swee Hock School of Public Health, National University of Singapore, Singapore, Singapore. ${ }^{3}$ Department of Epidemiology, School of Public Health, University of California Los Angeles, Los Angeles, USA. ${ }^{4}$ Department of Infectious Diseases, Communicable Disease Centre, Tan Tock Seng Hospital, 11 Jalan Tan Tock Seng, Singapore. ${ }^{5}$ Department of Medicine, Yong Loo Lin School of Medicine, National University of Singapore, Singapore, Singapore.

Received: 11 September 2012 Accepted: 31 October 2012

Published: 6 November 2012

\section{References}

1. Chaix $C$, et al: Control of endemic methicillin-resistant Staphylococcus aureus: a cost-benefit analysis in an intensive care unit. JAMA 1999, 282(18):1745-1751.

2. Cosgrove SE, et al: The impact of methicillin resistance in Staphylococcus aureus bacteremia on patient outcomes: mortality, length of stay, and hospital charges. Infect Control Hosp Epidemiol 2005, 26(2):166-174.

3. Rubin RJ, et al: The economic impact of Staphylococcus aureus infection in New York City hospitals. Emerg Infect Dis 1999, 5(1):9-17.

4. Kluytmans J, van Belkum A, Verbrugh H: Nasal carriage of Staphylococcus aureus: epidemiology, underlying mechanisms, and associated risks. Clin Microbiol Rev 1997, 10(3):505-520.

5. Wertheim HF, et al: The role of nasal carriage in Staphylococcus aureus infections. Lancet Infect Dis 2005, 5(12):751-762.

6. Davis KA, et al: Methicillin-resistant Staphylococcus aureus (MRSA) nares colonization at hospital admission and its effect on subsequent MRSA infection. Clin Infect Dis 2004, 39(6):776-782.

7. Huang SS, Platt R: Risk of methicillin-resistant Staphylococcus aureus infection after previous infection or colonization. Clin Infect Dis 2003, 36(3):281-285.

8. Harbarth S, Fankhauser C, Schrenzel J, et al: UNiversal screening for methicillin-resistant staphylococcus aureus at hospital admission and nosocomial infection in surgical patients. JAMA 2008, 299(10):1149-1157.

9. Drapeau CM, et al: Role of previous hospitalization in clinically-significant MRSA infection among HIV-infected inpatients: results of a case-control study. BMC Infect Dis 2007, 7:36.

10. Gupta $\mathrm{K}$, et al: Trends in prescribing beta-lactam antibiotics for treatment of community-associated methicillin-resistant Staphylococcus aureus infections. J Clin Microbiol 2007, 45(12):3930-3934.

11. Haley CC, et al: Methicillin-resistant Staphylococcus aureus infection or colonization present at hospital admission: multivariable risk factor screening to increase efficiency of surveillance culturing. J Clin Microbiol 2007, 45(9):3031-3038.

12. Klevens RM, et al: Community-associated methicillin-resistant Staphylococcus aureus and healthcare risk factors. Emerg Infect Dis 2006, 12(12):1991-1993.

13. Tumbarello $\mathrm{M}$, et al: Risk factors and predictors of mortality of methicillinresistant Staphylococcus aureus (MRSA) bacteraemia in HIV-infected patients. J Antimicrob Chemother 2002, 50(3):375-382.

14. Onorato $\mathrm{M}$, et al: Risk factors for colonization or infection due to methicillin-resistant Staphylococcus aureus in HIV-positive patients: a retrospective case-control study. Infect Control Hosp Epidemiol 1999, 20(1):26-30.

15. Villacian JS, et al: Prevalence of and risk factors for nasal colonization with Staphylococcus aureus among human immunodeficiency virus-positive outpatients in Singapore. Infect Control Hosp Epidemiol 2004, 25(5):438-440

16. Hidron Al, et al: Risk factors for colonization with methicillin-resistant Staphylococcus aureus (MRSA) in patients admitted to an urban hospital: emergence of community-associated MRSA nasal carriage. Clin Infect Dis 2005, 41(2):159-166.

17. Senthilkumar A, Kumar S, Sheagren JN: Increased incidence of Staphylococcus aureus bacteremia in hospitalized patients with acquired immunodeficiency syndrome. Clin Infect Dis 2001, 33(8):1412-1416.

18. Nguyen MH, et al: Nasal carriage of and infection with Staphylococcus aureus in HIV-infected patients. Ann Intern Med 1999, 130(3):221-225. 
19. Esuvaranathan K, et al: A study of 245 infected surgical wounds in Singapore. J Hosp Infect 1992, 21(3):231-240.

20. Hsu LY, et al: Antimicrobial drug resistance in Singapore hospitals. Emerg Infect Dis 2007, 13(12):1944-1947.

21. Sen P, et al: Rising trends of STIs and HIV infection in Singapore- a review of epidemiology over the last 10 years (1994 to 2003). Ann Acad Med Singapore 2006, 35(4):229-235.

22. Mertz $D$, et al: Throat swabs are necessary to reliably detect carriers of Staphylococcus aureus. Clin Infect Dis 2007, 45(4):475-477.

23. Wertheim HF, et al: Low prevalence of methicillin-resistant Staphylococcus aureus (MRSA) at hospital admission in the Netherlands: the value of search and destroy and restrictive antibiotic use. J Hosp Infect 2004, 56(4):321-325.

24. Chow A, et al: Universal methicillin-resistant Staphylococcus aureus (MRSA) screening: comparison of anatomic screening sites for patients with high and low prevalence of MRSA carriage. Infect Control Hosp Epidemiol 2012, 33(3):315-317.

25. Myskowski PL, Straus DJ, Safai B: Lymphoma and other HIV-associated malignancies. J Am Acad Dermatol 1990, 22(6 Pt 2):1253-1260.

26. Czepiel J, et al: The case of a diffuse large B-cell lymphoma (DLBCL) in a course of HIV. HIV \&amp; AIDS Rev 2010, 9(1):22-25.

27. Bain BJ: Lymphomas and reactive lymphoid lesions in HIV infection Blood Rev 1998, 12(3):154-162.

28. Nguyen V, et al: Cutaneous T-cell lymphoma and Staphylococcus aureus colonization. J Am Acad Dermatol 2008, 59(6):949-952.

29. Tsambiras $P E$, et al: Infectious complications of cutaneous t-cell lymphoma. Cancer Control 2001, 8(2):185-188.

30. McDonald LC, et al: Colonization of HIV-infected outpatients in Taiwan with methicillin-resistant and methicillin-susceptible Staphylococcus aureus. Int J STD AIDS 2003, 14(7):473-477.

31. Ramsetty SK, et al: Risks for methicillin-resistant Staphylococcus aureus colonization or infection among patients with HIV infection. HIV Med 2010, 11(6):389-394.

32. Hollis RJ, et al: Familial carriage of methicillin-resistant Staphylococcus aureus and subsequent infection in a premature neonate. Clin Infect Dis 1995, 21(2):328-332.

33. Hicks NR, Moore EP, Williams EW: Carriage and community treatment of methicillin-resistant Staphylococcus aureus: what happens to colonized patients after discharge? J Hosp Infect 1991, 19(1):17-24.

34. Reilly JS, et al: Universal screening for meticillin-resistant Staphylococcus aureus in acute care: risk factors and outcome from a multicentre study. J Hosp Infect 2012, 80(1):31-35.

35. Popovich KJ, et al: Community-associated methicillin-resistant Staphylococcus aureus colonization in high-risk groups of HIV-infected patients. Clin Infect Dis 2012, 54(9):1296-1303.

36. Szumowski JD, et al: Methicillin-resistant Staphylococcus aureus colonization, behavioral risk factors, and skin and soft-tissue infection at an ambulatory clinic serving a large population of HIV-infected men who have sex with men. Clin Infect Dis 2009, 49(1):118-121.

doi:10.1186/1742-6405-9-33

Cite this article as: Kyaw et al.: Prevalence of and risk factors for MRSA colonization in HIV-positive outpatients in Singapore. AIDS Research and Therapy 2012 9:33.

\section{Submit your next manuscript to BioMed Central and take full advantage of:}

- Convenient online submission

- Thorough peer review

- No space constraints or color figure charges

- Immediate publication on acceptance

- Inclusion in PubMed, CAS, Scopus and Google Scholar

- Research which is freely available for redistribution 